\title{
ARTERIAL BLOOD FLOW PATTERNS IN HUMAN SUBJECTS AND THEIR EFFECT ON INDICATOR DILUTION CURVES FROM VARIOUS ARTERIAL SITES *
}

\author{
By RAMON L. LANGE, CARLISLE SMITH AND HANS H. HECHT \\ (From the Departments of Medicine and Radiology, University of Utah College of Medicine, \\ Salt Lake City, Utah)
}

(Submitted for publication March 11, 1960; accepted May 13, 1960)

Indicator dilution curves obtained from various systemic arterial sampling sites are commonly used for analysis of blood flow, cardiac shunts and valvular regurgitation, and for study of segments of the blood volume. It is generally assumed that the contour of such curves is largely determined by the passage and dilution of the indicator in the central circulation and that changes occurring during arterial passage are minor and predictable on the basis of the expected velocity of arterial blood flow and the distances of the commonly used sampling sites from the aortic root. Although it is accepted that quantification of blood flow is unaffected by changes in curve contour so long as the first circulation of indicator can be identified, the estimation of other parameters available from curve analysis may be significantly influenced by the validity of the above assumption. Beard, Wood and Clagett (1) and Fox, Sutterer and Wood (2) have demonstrated considerable differences in contour as well as appearance time of curves obtained from the femoral (FA), radial (RA) and dorsalis pedis arteries. The first study showed delay in the FA components in coarctation of the aorta as compared with the RA curve, whereas the latter study noted delay and skewing of the RA or pedal curve as compared with the FA curve. The FA curves were similar in contour to the abdominal aortic curves in normal subjects. During preliminary studies of an investigation which attempted to quantitate valvular regurgitation by comparison of indicator dilution curves recorded simultaneously from the pulmonary artery (PA) and a systemic artery, we noted that in normal subjects only FA curves consistently resembled the PA curve while those recorded from the

* Supported in part by grants from the Utah Heart Association and the United States Public Health Service (Grant no. H-3126 and HTS 5150). brachial artery (BA), or RA frequently showed unpredictable deviations in curve parameters.

Although these studies clearly indicate that the sampling site as well as the sampling system (3-6) play an important role in the parameters of dilution curves, it is not clear whether the effects of arterial passage are a predictable characteristic in each arterial system and are constant from subject to subject and from moment to moment, or whether capricious effects occur which are related to spontaneous or reflex local disturbances, possibly produced by the sampling technique.

The present study was undertaken in an attempt to define the extent of variation in curves as obtained from commonly employed arterial sites and to examine the possible mechanism for distortion or skewing of the curves. The results of human studies led to the parallel investigation in a hydraulic analog system having three characteristics considered most important in determining the presence and extent of curve alteration: pulsatile flow, a branched elastic system, and variable efflux resistances. Although the effects on a dilution curve of constant flow through rigid tubing are well known (3-6), little evidence exists as to the pulsatile flow through a branched elastic system encountered in man.

\section{METHODS}

Human studies. Studies were carried out in 12 human subjects in the resting state during the course of venous cardiac catheterization. Simultaneous FA and BA curves were obtained following right heart or venous injection of $\mathrm{T}-1824$ or indocyanine green in 4 normal subjects. In 3 others, simultaneous RA and FA curves were obtained (Table I, Subjects 1 through 7). Three patients with coarctation of the aorta were examined: one with minimal narrowing showing only alteration of the central pressure slope (Patient 8), and two with evidence of severe constriction of the aorta and well developed collateral circulation (Patients 9 and 10). An- 
TABLE I

Parameters of simultaneous dilution curves from two arterial sites*

\begin{tabular}{|c|c|c|c|c|c|c|}
\hline $\begin{array}{l}\text { Subject } \\
\text { no. }\end{array}$ & Age, Sex & Diagnosis & $\underset{\text { sites } \dagger}{\text { Sampling }}$ & $\Delta t_{\mathrm{a}} \ddagger$ & $\Delta t_{p} \ddagger$ & $\Delta\left(t_{\mathrm{d}-}-t_{\mathrm{a}}\right) \ddagger$ \\
\hline $\begin{array}{l}1 \\
2 \\
3 \\
4 \\
5 \\
6 \\
7\end{array}$ & $\begin{array}{l}21, M \\
18, M \\
30, M \\
36, M \\
29, M \\
23, M \\
29, M\end{array}$ & $\begin{array}{l}\text { Normal } \\
\text { Normal } \\
\text { Normal } \\
\text { Normal } \\
\text { Normal } \\
\text { Normal } \\
\text { Repaired }\end{array}$ & $\begin{array}{l}\text { FA/BA } \\
\text { FA/BA } \\
\text { FA/BA } \\
\text { FA/BA } \\
\text { FA/RA } \\
\text { FA/RA } \\
\text { FA/RA }\end{array}$ & $\begin{array}{r}+2.3 \\
+2.0 \\
+1.0 \\
0.0 \\
+3.5 \\
+6.0 \\
+3.0\end{array}$ & $\begin{array}{r}+3.5 \\
+4.0 \\
+1.5 \\
0.0 \\
+4.7 \\
+8.5 \\
+3.0\end{array}$ & $\begin{array}{r}+5.5 \\
+6.0 \\
+1.0 \\
+1.0 \\
+6.0 \\
+10.5 \\
0.0\end{array}$ \\
\hline 8 & $24, \mathrm{M}$ & $\begin{array}{l}\text { atrial septal defect } \\
\text { Funct. } \\
\text { coarctation } \\
\text { aorta }\end{array}$ & $\mathrm{FA} / \mathrm{BA}$ & +1.0 & +1.5 & +1.0 \\
\hline $\begin{array}{r}9 \\
10 \\
11 \\
12\end{array}$ & $\begin{array}{l}38, M \\
19, M \\
66, M \\
23, \mathrm{~F}\end{array}$ & $\begin{array}{l}\text { Severe } \\
\text { Moderate } \\
\text { Abd. aortic } \\
\text { Bradycardia }\end{array}$ & $\begin{array}{l}\mathrm{FA} / \mathrm{BA} \\
\mathrm{FA} / \mathrm{BA} \\
\mathrm{FA} / \mathrm{BA} \\
\mathrm{FA} / \mathrm{BA}\end{array}$ & $\begin{array}{r}-1.0 \\
-1.0 \\
-8.0 \\
0.0\end{array}$ & $\begin{array}{r}-2.0 \\
-1.5 \\
-9.0 \\
0.0\end{array}$ & $\begin{array}{r}-5.0 \\
-1.0 \\
-2.0 \\
0.0\end{array}$ \\
\hline
\end{tabular}

* Records were obtained by using either two direct recording oximetergalvanometer systems or two densitometergalvanometer systems (see Methods).

$\dagger$ FA, BA and RA refer to curves obtained from the femoral artery, brachial artery and radial artery. In Subject 9, the left brachial artery was used.

$\pm t_{a}, t_{p}$ and $t_{d}-t_{s}$ refer to appearance time, peak concentration time, and passage time, respectively, following the nomenclature proposed by others (2). $\Delta t_{\mathrm{a}}, \Delta \mathrm{t}_{\mathrm{p}}$ and $\Delta\left(\mathrm{t}_{\mathrm{d}}-\mathrm{t}_{\mathrm{a}}\right)$ refer to the relationship between values of the two simultaneously recorded curves. Positive sign indicates that FA curve measurements were the smaller.

other subject (Patient 11) had a large abdominal aortic aneurysm and Patient 12 was included because of a bradycardia of 36 beats per minute. FA and BA curves were obtained in Patients 8 through 12.

Arterial punctures were performed following liberal infiltration of the puncture site with 2 per cent procaine; 20 gauge needles were used for the radial and 18 gauge or 20 gauge needles for the brachial and femoral punctures. None of the patients complained of discomfort from the indwelling needle. The device for simultaneous sampling from two sites is described elsewhere. A sampling rate of 0.3 to $0.6 \mathrm{ml}$ per second was used in all studies. Cuvet oximeters ${ }^{1}$ or cuvet densitometers ${ }^{2}$ used were attached directly to the arterial needles, and the delay times due to the dead space of cuvet tubing $(0.5 \mathrm{ml}$ in the oximeter and $0.25 \mathrm{ml}$ in the densitometer) were equal and less than one second. The curves were recorded by a kymographic camera ${ }^{3}$ at a paper speed of $5 \mathrm{~mm}$ per second. The downslopes were extrapolated to points of equal concentration.

Paired curves were analyzed for the following parameters: appearance time, $t_{a}$, and peak concentration time, $t_{p}$. The "disappearance time" was estimated by extrapolating the curve with the most rapidly decaying concentration to 0.05 of the concentration at the beginning of apparent exponential decrease in concentration. The other curve is then extrapolated to an equal concentration. This is taken to be the time when the two sampling

1 Wood cuvet oximeter, Waters Corp., Rochester, Minn. 2 XC-100A densitometer, Waters Corp., Rochester, Minn.

3 Waters kymographic camera, model G-12, Waters Corp., Rochester, Minn. sites reflect a common event occurring upstream in the aorta.

In addition to the experiments described above, arterial blood flow patterns were studied in 8 normal subjects and in 3 individuals with valvular heart disease who were free from obvious arterial disease. This was accomplished by observing with cinefluorography ${ }^{4}$ the distribution and flow of 50 per cent diatrizoate sodium $(\mathrm{Hy}-$ paque) introduced into an artery. Following percutaneous puncture with an 18 gauge needle, a specially modified 20 gauge needle, $10 \mathrm{~cm}$ long with the tip occluded and fenestrations placed radially over the distal centimeter of length was passed through the no. 18 needle so that the multiple openings lay within the lumen of the vessel studied. After free flow was ascertained, cinefluorographic recordings were made of the streaming pathways of slowly injected, $1 \mathrm{ml}$ per second, and rapidly injected, 2 to $4 \mathrm{ml}$ per second, 50 per cent Hypaque. In 4 subjects injections were made into the brachial artery. The femoral artery was used in 4 normals and in 3 patients with mild aortic valvular incompetence. In 3 of the normal subjects in whom FA injection was made, records were obtained before and after occlusion of arterial blood flow just above the knee by means of a pneumatic cuff inflated to suprasystolic pressure.

Model studies. The hydraulic analog consisted of a simple branched system of rubber tubing with a small glass " $Y$ " tube at the bifurcation. Water was pumped through the system by means of a Sigma Motor finger pump, which delivered pulsatile flow with a stroke volume about $10 \mathrm{ml}$ at a rate of 80 per minute. The volume of the undistended system measured, under various con-

\footnotetext{
${ }^{4}$ Cinefluorex, Westinghouse Corp.
} 
ditions, from 16 to $20 \mathrm{ml}$. The distal ends of the two branches of tubing could be controlled by screw clamps to provide variable outflow resistances. Indicator was injected proximal to the pump. The latter provided adequate mixing. Dilution curves were obtained through short no. 20 needles connected to direct recording cuvet oximeters. Sampling was accomplished by the paired sampling syringes mentioned previously (3). The rate of sampling at all times was less than 5 per cent of the flow through the model. The model was intended to closely simulate the relative capacity of the arterial tree and the flow rate through it. The following paragraph describes the manner in which local alterations in flow were simulated.

The following experimental conditions were set up to test their influence on the contour of a dilution curve: a) sampling from two branches having equal flow, but equal or unequal volume; $b$ ) sampling from two branches having unequal flow, accomplished by changing outflow resistance of one branch only; $c$ ) sampling from proximal and distal segments of one branch having increased outflow resistance; $d$ ) sampling from a nonexpansile system made so by lowering outflow resistance in both branches; $e$ ) sampling from both branches with elevated, but slightly unequal, outflow resistances.

The failure of the galvanometer beam to return to the baseline after curve inscription deserves mention. It was noted that the polyethylene tubing, leading water and $\mathrm{T}-1824$ dye through the recording cuvet, would remain faintly tinted with blue color long after the effluent was

A

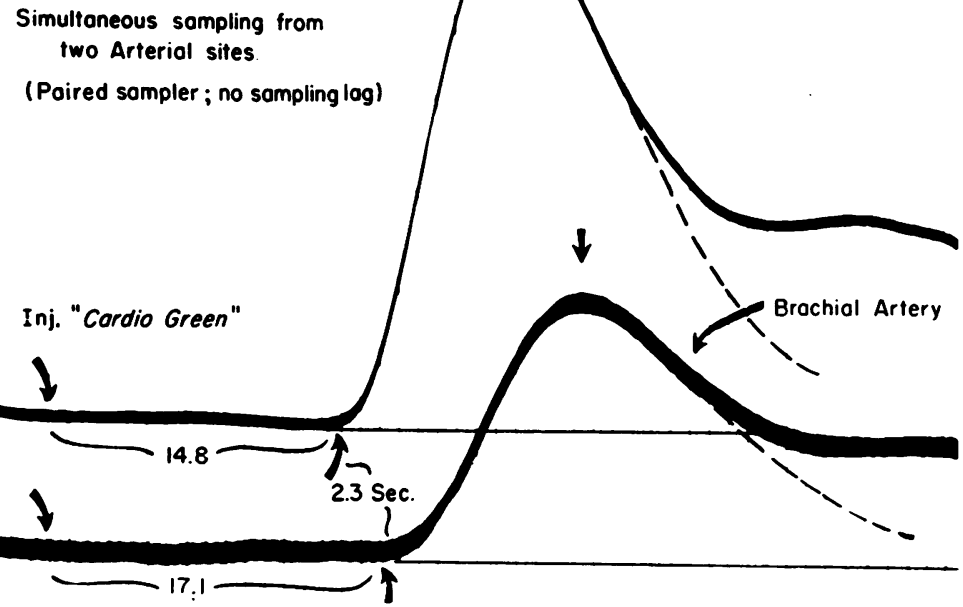

B

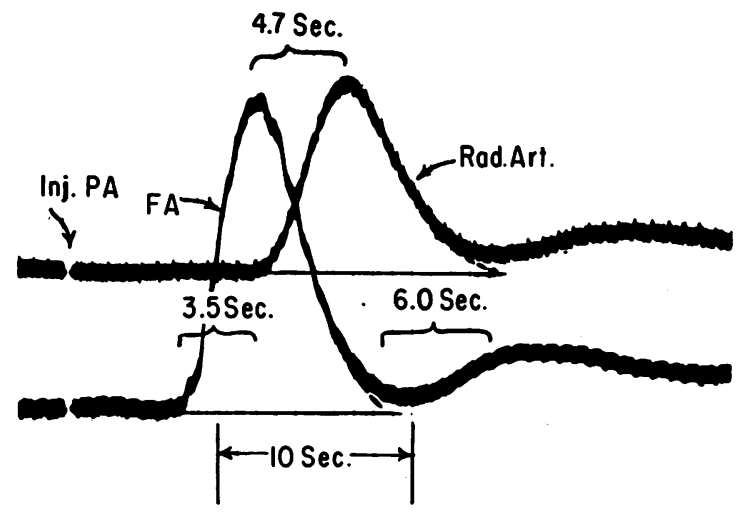

Fig. 1. A. A characteristic SET of CuRves from a NORMal Male (SUbJECT 1, TABLE I) SHOWING FAIRLY EXTREME VARIATION BETWEEN BA AND FA curves. Curves are extrapolated to equal concentrations. B. Simultaneous CURVES From the FA ANd RA following PA injection. Recordings were made using cuvet densitometers sensitive to indocyanine green. The variation of the RA curve from the FA curve is qualitatively the same as the variation of the BA curve from the FA curve in $1 \mathrm{~A}$. 


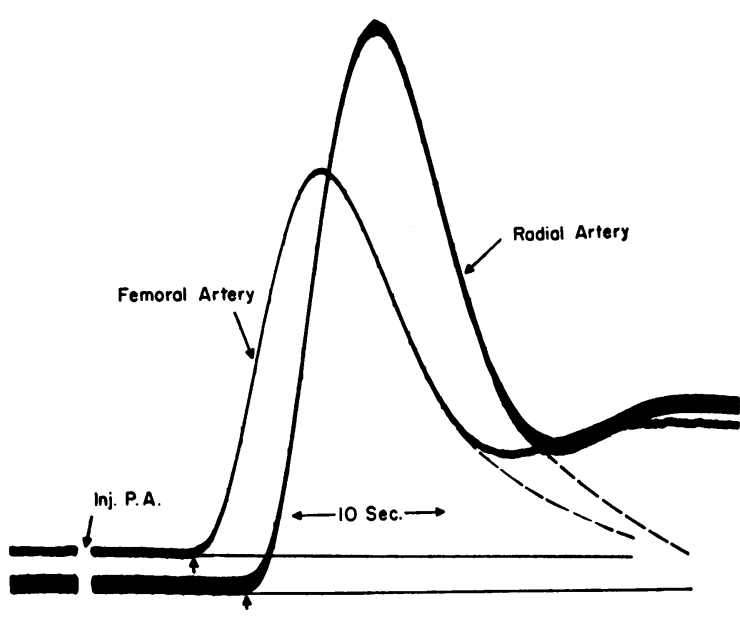

Fig. 2. Curves from FA and RA in a 29 year old male (Subject 5, Table I) following successful RePAIR of AN ATRIAL SEPTAL DEFECT. Note the delay of all parameters of the RA curve without skewing.

colorless. We conclude that loose binding between the dye and plastic occurred with a significant effect only at low concentration. We, therefore, doubt the accuracy of the downslope at lower concentrations and have used the segment of the curve shortly after the peak for comparative purposes.

\section{RESULTS}

\section{Comparison of human curves obtained simul-} taneously from two arteries. As demonstrated in Figures 1, 2 and 3, considerable variability is encountered when two curves are recorded simultaneously from various arterial sites following single venous or right heart injection.

The measured parameters of paired curves are listed in Table I. There is frequently not only de-
Coorctation of Aorto

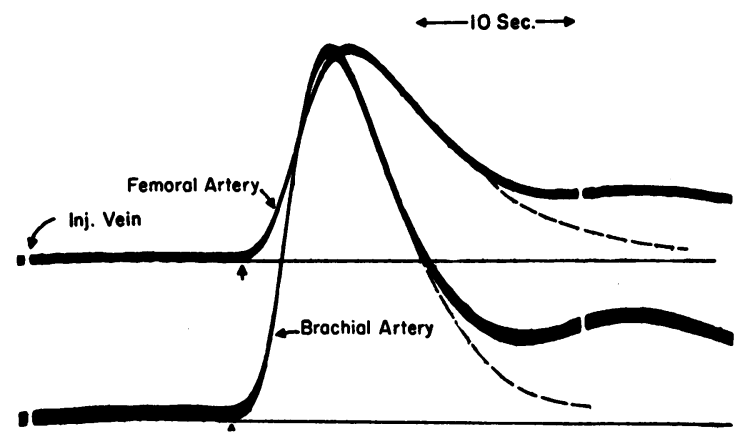

Fig. 3. The efFects of SEVERE Mechanical obStrucTION OF AORTA DISTAL TO THE LEFT SUBCLAVIAN ARTERY (SAMPLING FROM THE LEFT BRACHIAL ARTERY). The delay and skewing of the FA curve as compared to the BA curves is probably the result of increased $U / F$ ratio distal to the coarctation and to the presence of numerous collaterals with variable transit times.

layed appearance, $t_{\mathrm{a}}$, of dye at the BA or RA when compared with the FA, but in addition the curve is skewed by further delay in the peak concentration time, $t_{p}$, and prolongation of the passage time, $t_{d}-t_{a}$ (Subjects 1, 2, 5 and 6). These findings agree in general with those of Fox, Sutterer and Wood (2), but there is not always significant alteration of the BA curves (Subjects 3, 4, 8 and 12) and the RA curve may be delayed without other alteration (Subject 7). Examination of the pair of curves in Figure 1A shows that the delay in $t_{a}$ is about 2.3 seconds, the delay in $t_{p} 3.5$ seconds and the increase in $t_{d}-t_{a}$ is even greater, 5 seconds. Thus, the first appearing dye is delayed at the BA site, but not so much as is the dye par-

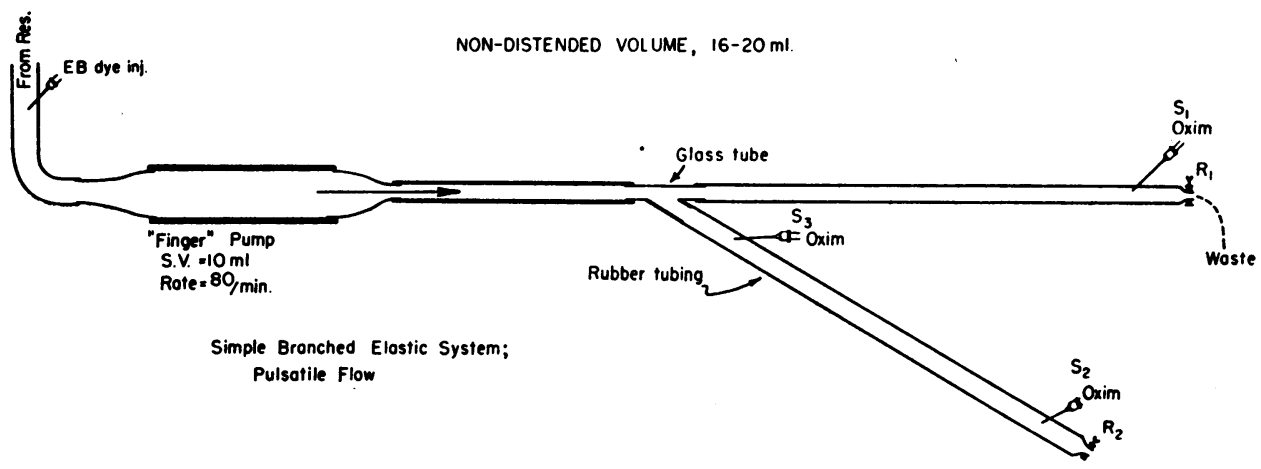

Fig. 4. The outline of the model of a single BRANChed System with EXPANSile and PULSATILE FLOW. Ratio of "diastolic" volume to stroke volume and pump rate are close to these values expected in the human arterial tree. $R$ and $R_{2}$ are variable resistances; $S_{1}, S_{2}$ and $\mathrm{S}_{\mathbf{8}}$ are sampling sites. 


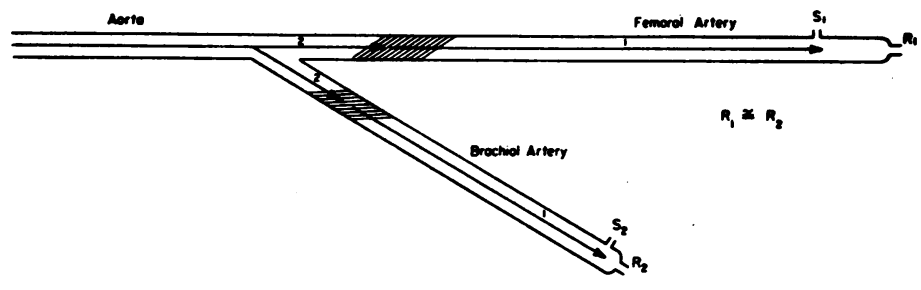

Equal length, diameter and flow

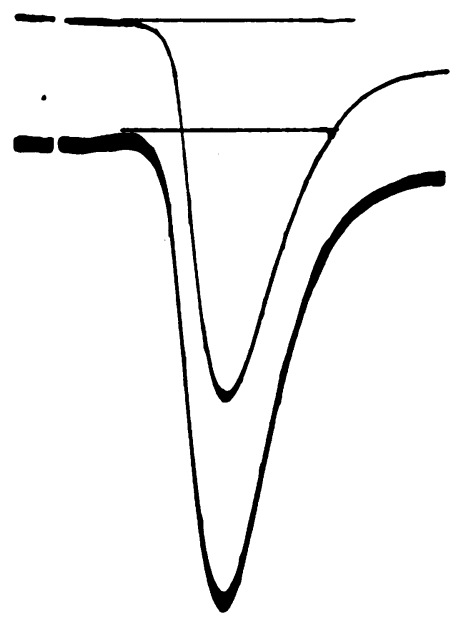

Unequal length, diometer,and Equal flow

Fig. 5. Two successive STROKe volumes From a Dilution CURVe, Each STROKE A RATHER HOMOGENEOUS "STEP FUNCTION" OF CONCENTRATION. The shaded areas indicate that the mixing length is short compared to the distance over which concentration tends to be constant. This concept would explain the finding of nearly constant contour of curves from various portions of a system where $U / F$ ratios were equal in the two branches. Below, representative curves from such a system are shown.

ticle with average transit time, $\overline{\mathrm{t}}$, and even less than the particles near $t_{d}$. Figure $1 B$, recorded with more rapidly responding cuvet densitometers from the FA and RA, shows similar findings. The cases included in Table $I$ represent all possible combinations of $\mathrm{FA}$ and $\mathrm{BA}$ and $\mathrm{RA}$ relationships: a) the common occurrence of delayed appearance and skewness of the BA with respect to the FA (Subject 1 and 2 ); $b$ ) no significant difference between FA and BA curves (Subjects 3, 4, 8 and 12$) ; c$ ) delay in onset, $t_{a}$, of the RA curves with or without variation of contour (Subjects 5, 6 and 7$) ; d$ ) reversal of usual findings in severe coarctation (Subjects 9 and 10) and in abdominal aortic aneurysm (Subject 11).

2. Cinefluorex studies. In all FA studies including those of three subjects with mild aortic regurgitation, Hypaque, injected slowly at $1 \mathrm{ml}$ per second, was carried distally without visible diastolic retrograde movement. During repeated studies, or if Hypaque was injected at a faster rate for 8 to 10 seconds, a variable degree of retrograde movement, never more than 3 to $5 \mathrm{~cm}$, was apparent in diastole. There was always rapid clearance of injectate from the site.

In the three FA studies with cuff occlusion of the arterial flow just above the knee, striking retrograde flow of Hypaque was seen as far proximal as the aortic bifurcation. Subsequent injections with the cuff released showed the relationships seen in the control period.

Brachial artery injections revealed a more variable pattern. In one patient there was no visible retrograde movement. In two remaining normal 
subjects, there was a variable diastolic retrograde movement of injectate with slow clearing of indicator after cessation of injection. In one normal subject, a brief rapid injection of a small volume resulted in retrograde flow so striking that the subclavian artery was opacified and, although radial pulse was palpable, washout time was prolonged to 18 seconds after cessation of injection.

3. Model studies. The outcome of Studies 1 and 2 above suggested the simple branched elastic model (Figure 4) with pulsatile flow and the variations of the volume-flow relations described above. When equal flow occurs through equal volumes of the pulsatile system, the curves obtained from each branch are identical in all parameters. When the volumes to be traversed are unequal but the flow rates are identical, the similarity of the two curves is maintained, but of course the distal curve has delayed inscription (Figure 5). Thus, differences in volume con- tained in the two branches of the system do not significantly change the contour of the curves. However, a disproportionate decrease in flow of one branch ("vasoconstriction"), with volume, elasticity and stroke volume kept constant, shows that, in addition to the expected delay in the appearance time, the peak concentration time and the extrapolated passage time are further significantly delayed (Figure 6). In fact, when compared to the delay in $t_{a}, t_{p}$ and $t_{d}$ show even greater "smearing," i.e., they demonstrate progressive delay when compared to the same measurement of the branch of the model that had normal outflow resistance. This same relative difference between $t_{a}, t_{p}$ and $t_{d}$ was noted in many human subjects when simultaneous sampling from radial or brachial artery was compared with the femoral artery.

With high outflow resistance of one branch of the model, similar differences appear when curves are recorded simultaneously from the proximal

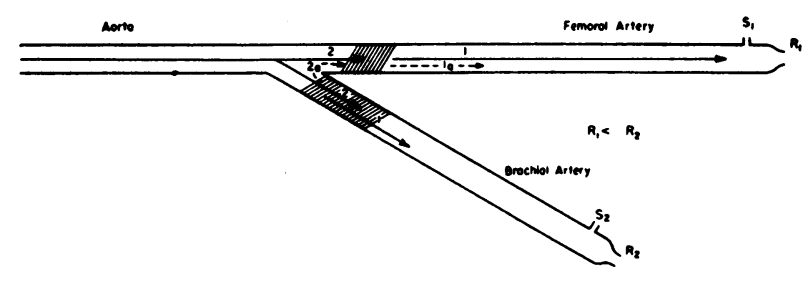

Unequal length, diameter and moderately unequal flow. Resistonce $L>S$

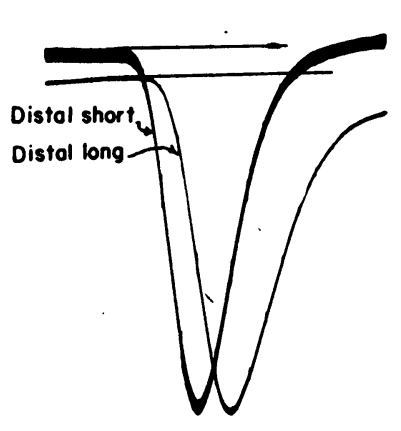

Unequal flow, length ond diameter Resistonce $L>>S$

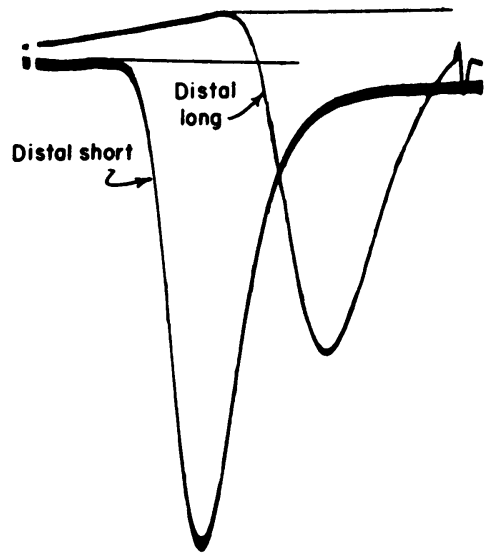

Fig. 6. Schematic Representation of INCREASEd U/F IN A SIDE BRANCH ACCOMPLISHED BY INCREASING RESISTANCE $R_{2}$. Now the mixing length is an appreciable fraction of the total segment occupied by the aliquot of stroke volume. Below are seen the effects of two conditions of unequal flow. 

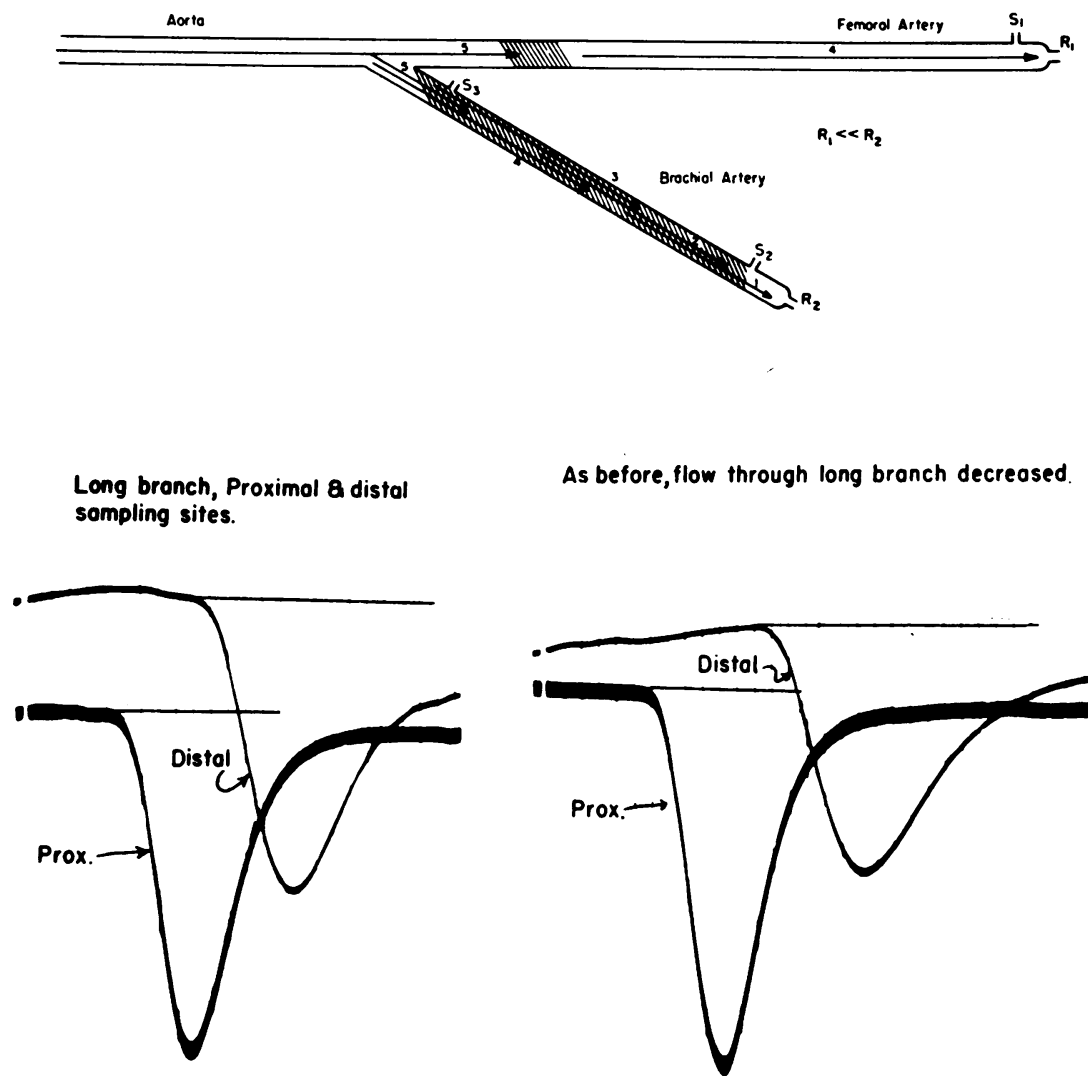

As before, flow through long bronch decreosed.

Long bronch, Proximal 8 distal

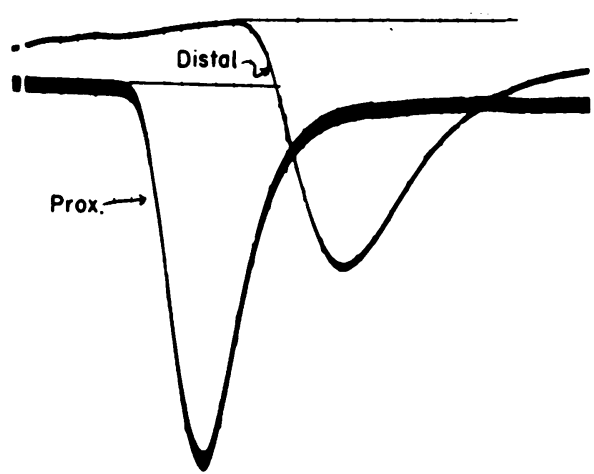

Fig. 7. The Depiction of Mixture of SEVERAL aliquots of SUCCEssive STROKes IN THE HIGH U/F SIDE BRANCH. Thus the fourth stroke concentration may be recorded from the FA (or $S_{3}$ ) while the first stroke concentration reaches the site $S_{2}$. Strokes 1 through 5 intermingle, strokes 1 and 2 mix "ahead" with undyed blood and will be recorded in advance of the centroid of the curve. After $t_{p}$, this process is reversed with volumes of lower concentration entering the segment and mixing with higher concentrations present; in consequence delayed clearance occurs. Note the similarity of curves from $S_{3}$, the proximal site on the side branch, and $S_{1}$ of Figure 6.

and the distal end of the same branch (Figure 7). The proximal curve is similar to a record obtained from a branch with normal outflow resistance; the distal curve is similar to that from the constricted branch of Figure 6, so that the difference in curve parameters sampled from these two sites of the same "vasoconstricted" branch is essentially the same as the difference obtained from one normal and one constricted branch. This is more marked when the resistance in the sampled tube is further increased.

Finally, conditions of flow in a non-expansile system were simulated by causing such low outflow resistance that discontinuous (systolic) rather than continuous but pulsatile flow occurred. This solely systolic and presumably laminar flow did not cause distention of the rubber tubing and no appreciable elastic effect remained (Figure 8 left). Under these circumstances no distortion of contour was found. Likewise, when extreme, although unequal increase in resistance was applied to the two branches, there again was no distortion of proximal and distal curves obtained from one branch (Figure 8 right).

\section{DISCUSSION}

From the results obtained in the human studies, it is apparent that, in the absence of structural abnormality of the aorta, the FA curve invariably has values of $t_{a}, t_{p}$ and $t_{d}-t_{a}$ equal to or less than those of a simultaneous curve from another arterial site. Published curves of Fox and associates (2) indicate that the FA curve does not differ significantly from the curve obtained from the 
abdominal aorta except for the expected brief and equal delay of all parameters. Comparison of curves obtained from the PA and FA following venous injection in normal subjects shows only a small, nearly constant alteration in contour (7). These observations support the conclusion that, when dealing with a normal arterial tree, the FA curve may be considered a close representative of its antecedent as it emerged from the left ventricle. The reason this site is unique among those commonly utilized may be the nearly pure forward flow patterns generally found with FA radiographic studies, except when mechanical obstruction is induced distal to the site studied. As a corollary, of all commonly used sites in man, the FA curve can be recorded with a minimum of delay and is not likely to imply a "Stewart" volume greater than the anatomical volume existing between aortic valve and sampling site. This conclusion is not warranted if gross aortic disease exists.

Curves from the BA may or may not differ from the FA curve in an apparently unpredictable man- ner. This correlates with variability of flow patterns seen in BA injection studies. Although the number of paired curves from the FA and RA is small, the latter curves show the most striking alterations and this is in keeping with the finding of slow clearance of radiopaque substance distal to the BA injection site even though severe alterations of flow patterns were not seen at that site.

The manner in which curves may be altered by arterial passage would seem to depend on local variation of flow and volume relations. The observed facts implicate two mechanisms, both of which are the consequence of local vasoconstrictive action: local regurgitation (return flow) because systolic uptake exceeds the runoff capacity of the segmental vascular bed, and a smaller systolic uptake over a prolonged time period because of a decrease in the compliance of the segment of arterial bed involved.

\section{A suggested skewing mechanism}

As stated, any explanation advanced for the mechanisms by which curves may be altered dur-

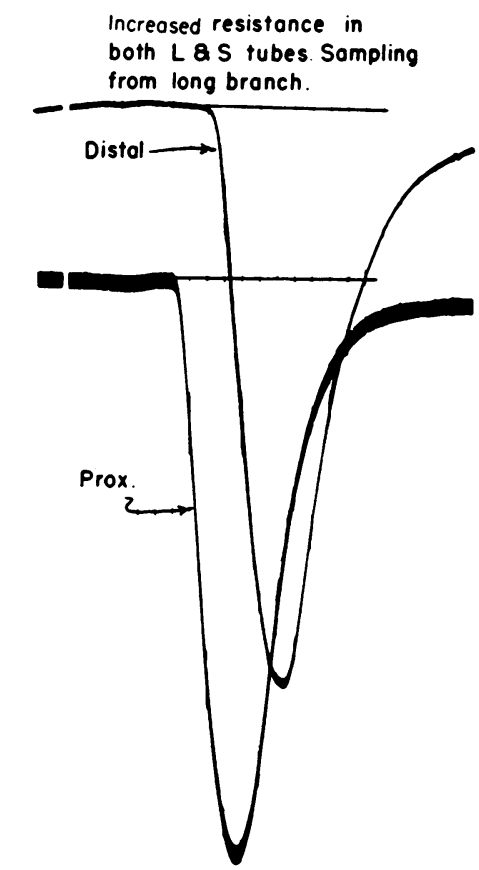

Fig. 8. (Left) The efFects of PUlsatile bUt NONEXPansile Flow. There is no discernible effect due to laminar flow in the side branch. (Right) EXtremely high RESistance in Both bRANCHES; PUlSATILE, EXpansile flow. Again only delay is seen in all portions of the curve from the distal site without skewing. This is similar to the curve from human subject, Figure 2. 
ing passage through the arterial tree must include considerations of a pulsatile elastic system. It must also embrace the premise that the normal arterial tree receives the dilution curve in successive stroke volumes of more or less homogeneous concentration which occupy segments along the tree which are long as compared to the distance over which longitudinal mixing can occur during the time of arterial passage. To be acceptable, longer mixing pathways, shorter length of constant concentration, longer time of passage, or a combination of these must be incorporated in the explanation. The following paragraphs and Figures 5 through 8 attempt this.

First, consider the various branches of the arterial tree as having a diastolic runoff equal to the net systolic uptake. If this ideal condition prevails, then all the blood entering a given branched segment in systole will eventually leave the appropriate drainage bed at the other end (Figure $5)$. To the extent that the ratio of systolic uptake or "capacitance" over diastolic runoff or flow $(\mathrm{U} / \mathrm{F})$ of a branched segment differs from the ideal situation, more or less blood will leave the drainage bed in diastole than the net amount which entered during systole. If the $\mathrm{U} / \mathrm{F}$ ratio is greater than average, then some of the systolic uptake which has penetrated the segment a certain distance will not be able to proceed and will flow or regurgitate into other segments with smaller $U / F$ ratios. This circumstance, illustrated in Figures 6 and 7, would allow a degree of regurgitation in any segment in which the $U / F$ ratio exceeded the average. The effects of this regurgitation on the remaining low $U / F$ vessels would not necessarily be discernible, since the concentration of indicator is identical in all of the systolic ejectate and subsequent diastolic runoff.

The effects of this localized regurgitation can be examined further. Let us consider the course of events which follows the end of systole. (Assuming the pressure is nearly the same at all points in this small segment, one may consider diastole to begin at nearly the same time in all parts.) Consider first the fate of the blood immediately adjacent to the arterioles; this will flow continuously forward during diastole and in this it is similar to all the juxta-arteriolar blood. However, the blood and indicator, which at the end of systole are found at the entrance to this segment, reverse flow and enter another drainage area. The fate of blood at various intermediate points will generally fall between these extremes and theoretically there is a point in such a segment where the two opposite effects balance and no net flow occurs during diastole. These reasonable conclusions thus far have not caused an alteration of the time concentration curve as it traverses this aberrant segment since, at any given instant, the concentration is the same in both average and aberrant segments. A second abnormal condition is needed for dispersion to occur and to result in different concentrations in the two segments at a given time. This may be related to the manner in which systolic "penetration" occurs. In any segment a certain end-diastolic volume exists. The systolic uptake is smaller than the end-systolic volume, and to the extent that the latter exceeds the former, more than one stroke will be required until the indicator substances have completely traversed a segment. If diastolic flow is normally related to net systolic uptake, i.e., equal $(U / F=1)$, then the average traversal time may be considered equal to the volume of the segment divided by the arteriolar runoff per second. It follows that with diminished runoff and constant volume, this time would increase inversely as the runoff decreased. Note that this will allow a delayed $t_{a}$ as seen in human and model curves, but not necessarily an alteration of the contour. This situation is encountered in Table I, Subject 7, and in Figures 2 and 8. A further essential is the distribution of small aliquots of each stroke volume (systolic ejection) over an additional volume (and between one another). Since it has been stipulated that the stability of the curve as normally seen is due to a short longitudinal mixing distance and the relatively much greater length of homogeneous concentration, it is possible to increase the dispersion and alter the curve either by decreasing the length of homogeneous concentration, by increasing the length of longitudinal exchange, or by allowing longer time for longitudinal mixing. When small aliquots of aortic ejectate enter a segment with increased ratio of $U / F$ so that several successive short subsegments have different concentrations, and if conditions within the segment allow a significant degree of longitudinal mixing, we finally have all three conditions necessary for definite alteration of the input curve. The cir- 
cumstances encountered in an "average" $\mathrm{U} / \mathrm{F}$ segment are shown schematically in Figure 5. It will be noted that there are long distances of homogeneous concentration and short distances over which mixing can occur. This situation is contrasted with a high ratio $\mathrm{U} / \mathrm{F}$ segment in which the distance between blood with two concentration levels may be of the same order as the distance of mixing (Figures 6 and 7).

Correlation of the theoretical mechanism outlined and the observed abnormalities follows. As successive small volumes of increasing concentration enter the segment of high $\mathrm{U} / \mathrm{F}$ and move more slowly, as in Figures 6 and 7, a delayed $\bar{t}$ is to be expected. However, the mixing effect will cause an apparent "advance" of the appearance time $t_{a}$, since it is delayed less than $\overline{\mathrm{t}}$; $\overline{\mathrm{t}}$ is not affected by the mixing process which is bidirectional and of equal magnitude. Since $t_{a}$ is delayed less than $\bar{t}$, the first part of the curve is longer than the simultaneous curve recorded from the undisturbed segment. After peak concentration is reached, and decreasing concentration occurs with successive strokes, the mixing of aliquots of several strokes now slows the clearance, i.e., $t_{d}-t_{p}$ is longer in the segment with a high $U / F$. The human studies, Figure $1 \mathrm{~A}$ and $\mathrm{B}$, and model studies, Figures 6 and 7 , are equivalent examples of this.

Of interest is the effect of mechanical obstruction on the contour of the dilution curve. Subject 8 (Table I), a patient with mild aortic coarctation, exhibited little functional evidence of obstruction (8) and showed the usual relations between FA and BA artery curves. Subject 9, with severe but incomplete obstruction, exhibited delay and skewing of the FA curve as compared to the BA curve, the reverse of the usual finding. This alteration of the FA curve is probably primarily caused by the increased $U / F$ ratio distal to the obstruction rather than by the presence of collateral pathways of variable transit times. This is suggested from the evidence that the alteration of the FA curve is similar to that seen in $B A$ when increased $U / F$ is suspected. Beard and co-workers (1) have shown that the delay of the FA curve is corrected following repair of the coarcted segment.

The theoretical concepts presented above fit the observed differences in human studies and receive support from cinefluorographic studies which have demonstrated the transformation of a normal bra- chial artery flow pattern to a "regurgitant" and almost stagnant flow pattern by local vasoconstrictive reactions. Model studies clearly show that, if there is no disproportion of $U / F$ allowed in the analog, the curve is nearly identical in all sites measured and the effects of laminar flow cannot be discerned. These methods of investigation also yielded results compatible with the theory that a curve may be altered by slow movement of a vascular column containing several different concentration levels where increased local mixing is possible. All of these are consequences of the increased $U / F$ ratio of that segment.

Although it has been convenient to consider certain portions of the peripheral arterial tree as extensions of "sampling system dead space" $(2,9)$, it is obvious from Table I that the effects of this type of dead space are quite variable and a single curve could not be analyzed by the approaches which Sherman, Schlant, Kraus and Moore (6) have applied to catheter sampling systems. For this reason, it is suggested that in the consideration of possible alteration of dilution curves only the predictable, measurable invariant "dead space" be used in corrective manipulation.

\section{SUM MARY}

Of all commonly used arterial sampling sites, the femoral artery is most likely to yield a curve which most closely approaches the curve as it emerges from the left ventricle and this curve will be recorded with the least delay. Variable alteration of one or more curve parameters was frequently seen when simultaneous brachial artery or radial artery curves were compared with those from the femoral artery. The distortion encountered may assume proportions which would lead to serious inaccuracy in the estimation of appearance time, mean circulation time and disappearance slope.

Radiopaque injections into the femoral and brachial arteries of human subjects without arterial disease show extremely variable flow patterns in the brachial artery but not in the femoral artery. This is apparently due to local vasoconstriction.

A simple branched model characterized by pulsatile flow in an elastic system, with dimensions of capacity and stroke volume proportional to those of the human large arterial bed, could duplicate all variations of dilution curves seen in the human 
studies by means of altered resistance to flow in the two branches.

In the absence of pulsatile, elastic flow in the model, alteration of the curves was not seen, thus indicating that the parameters measured were insensitive to the effects of laminar flow and longitudinal mixing.

It is concluded that whenever possible the femoral artery in humans should be the most distal sampling site used when accurate reproduction of the contour of the curve and accurate estimation of the arterial volume included in the "Stewart" or "needle to needle" volume is required.

A high degree of coarctation of the aorta and presumably obstructive disease of the great vessels or aneurysmal dilatation of the thoracic or abdominal aorta render the femoral artery curve invalid for accurate applications of a dilution curve.

\section{REFERENCES}

1. Beard, E. F., Wood, E. H., and Clagett, O. T. Study of hemodynamics in coarctation of the aorta using dye dilution and direct intra-arterial pressure recording methods. J. Lab. clin. Med. 1951, 38, 858.

2. Fox, I. J., Sutterer, W. F., and Wood, E. H. Dynamic response characteristics of systems for continuous recording of concentration changes in flowing liquid. J. appl. Physiol. 1957, 11, 390.

3. Dow, P. Effects of a sampling system on dye curves (abstract). Physiologist 1958, 1, no. 4, 17

4. Sheppard, C. W. Mathematical considerations of indicator dilution techniques. Minn. Med. 1954, 37, 93.

5. Renkin, E. M. Separation of solutes in washout of cylindrical tubes. Fed. Proc. 1959, 18, 127.

6. Sherman, H., Schlant, R. C., Kraus, W. L., and Moore, C. B. A figure of merit for catheter sampling systems. Circulat. Res. 1959, 7, 303.

7. Lange, R. L., and Hecht, H. H. Quantitation of valvular regurgitation from multiple indicator-dilution curves. Circulation 1958, 18, 623.

8. Patel, D. J., Lange, R. L., and Hecht, H. H. The concept of functional coarctation of large blood vessels. Amer. J. Med. 1959, 26, 761.

9. Emanuel, R. W., Lacy, W. W., and Newman, E. V. Relative effects of heart chambers, lungs, and mitral insufficiency on the shape of indicator dilution curves. Circulat. Res. 1959, 7, 141. 